\title{
Genetically modified Caenorhabditis elegans may lead to inaccurate toxicity evaluation of mixtures
}

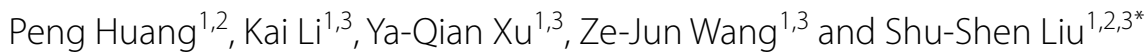

\begin{abstract}
Background: One of the major challenges in environmental science is how to assess the toxicity and risk of complex pollutant mixtures. However, only a few studies have pointed out that there is a significant difference between the toxicities of chemicals on genetically modified strains and wild-type organisms and there are few reports of the differences in the toxicity of chemical mixtures. Therefore, six chemicals, two substituted phenols (4-chlorophenol and 4-nitrophenol), two pesticides (dichlorvos and glyphosate) and two ionic liquids (1-butylpyridinium chloride and 1-butylpyridinium bromide), were selected to construct a six-component mixture system, the lethality of various mixtures on the genetically modified Caenorhabditis elegans strain mtl-2::GFP (MTL-2) at 12 and $24 \mathrm{~h}$ were determined, and differences in toxicity to other strains were compared.

Results: Although the toxicity of 4-chlorophenol on MTL-2 was not significantly different from that on N2 wild-type Caenorhabditis elegans (N2), the toxicities of the other five chemicals on MTL-2 were greater than those on N2. The toxicities of six single chemicals and nine mixture rays on MTL-2 increased with time, which is consistent with the effect on N2 and on the genetically modified strain sod-3::GFP Caenorhabditis elegans (SOD-3). The toxicological interactions of various mixtures in MTL-2 at $12 \mathrm{~h}$ were half antagonistic (ANT) and half additive (ADD), while at $24 \mathrm{~h}$, they were mainly synergistic (SYN). The toxicological interactions of various mixtures in MTL-2 change from ANT/ADD to primarily SYN with time, which is different from the change from ANT to ADD in SOD-3 and from SYN to ADD in N2.

Conclusions: The toxicity and toxicity interactions of chemical mixtures on different Caenorhabditis elegans strains are different. Therefore, it is necessary to examine the effect of genetic factors on the toxicological interaction of mixtures to avoid underestimating or overestimating the mixture risk.
\end{abstract}

Keywords: Time-dependent toxicity, Uniform design ray (UD-Ray), Mixture risk assessment, Concentration addition, Combination index

\section{Background}

With the rapid development of modern times, the number of chemicals contributing to anthropogenic contamination of the environment is increasing [1]. The increasing application of various chemicals often results

\footnotetext{
${ }^{*}$ Correspondence: ssliuhl@263.net

${ }^{1}$ Key Laboratory of Yangtze River Water Environment, College of Environmental Science and Engineering, Ministry of Education, Tongji University, 1239 Siping Road, Shanghai 200092, P. R. China

Full list of author information is available at the end of the article
}

in the formation of complex mixture pollutants. In reality, chemicals that usually occur at low concentrations and in mixtures will affect subtle physiological traits in organisms and may directly or indirectly cause longterm adverse ecological effects [2-6]. It is undeniable that humans are always exposed to complex mixtures; however, current risk assessments commonly focus on single chemicals or simple mixtures [7, 8]. Additionally, the mixture studied is often only a mixture ray $[9$, 10] in a multicomponent mixture system, which lacks representativeness and therefore does not reflect real 
environmental mixtures. It is necessary to rationally and effectively evaluate the combined toxicity of many mixtures with diverse concentration compositions [11]. The uniform design-based ray design (UD-Ray) developed in our laboratory $[8,12]$ is an effective method for the evaluation of combined toxicity. The UD-Ray can select some representative mixture rays from a number of mixtures for systematic investigation to reveal the toxicity of a mixture system [13-16].

The selection of model organism is often as important as mixture experimental design. Caenorhabditis elegans (C. elegans), due to its many features such as its short life cycle, ease of generating mass cultures and low cost, has been proven to be a sensitive and valuable bioindicator of toxicants $[17-20]$. In the face of the rapid development of transgenic technology, by introducing mutations into the green fluorescent protein (GFP) gene [21], C. elegans and genetically modified strains have also been used widely for ecological risk assessment and environmental toxicology [22-25]. For example, researchers evaluated the toxicity of heavy metal exposure and the possible transferable properties with GFP variants in C. elegans [26-29]. McVey et al. [30] studied three different strains with GFP-tagged neurons to facilitate visual assessment of neurons that were chronically exposed to glyphosate. Lenz et al. [31] used a daf-16::GFP transgenic strain to evaluate the potential toxicity of triclosan and triclocarban. A toxicity assessment of nanometer materials was performed in vivo using a GFP transgenic strain [32-34].

Our research group explored the toxicities and toxicological interactions of a six-component mixture system of two pesticides (dichlorvos (DIC) and glyphosate (GLY)), two substituted phenols (4-chlorophenol (4-CP) and 4-nitrophenol (4-NP)) and two ionic liquids (1-butylpyridinium chloride ([bpy]Cl) and 1-butylpyridinium bromide ([bpy $] \mathrm{Br}))$ on sod-3::GFP transgenic C. elegans (SOD-3) [35] and wild-type C. elegans (N2) [20]. It was shown that the toxicities of the six components were greater on genetically modified strains (SOD-3) than on $\mathrm{N} 2$. The toxicological interactions of various mixtures on SOD-3 and N2 were different. For example, at $12 \mathrm{~h}$, the interactions of most mixtures on SOD-3 were antagonistic (ANT) and those on N2 were synergistic (SYN) or additive (ADD). However, whether other genetically modified nematodes have similar toxicity characteristics and interaction patterns needs more in-depth study and more cases.

Since the expression of the metallothionein genes, $m t l$ 1 and $m t l-2$, is very low in wild-type C. elegans, the genes can significantly induce the transcriptional expression of metallothionein in intestinal tissues when C. elegans are exposed to heavy metals [36]. Based on this, the $m t l$ 2::GFP genetically modified C. elegans (MTL-2) could reveal the influence of genetically modified factors on the toxicities and toxicological interactions of nonmetallic mixtures. In our initial experiments, some nonmetallic compounds such as DIC and 4-CP exhibited obvious lethal toxicity to MTL-2 and had good concentrationresponse relationships, which implies that MTL- 2 can be a sensitive biomarker of lethal toxicity.

In this paper, using six chemicals DIC, GLY, 4-CP, 4-NP, [bpy]Cl and [bpy] Br as the mixture components to construct a six-component mixture system and using UD-Ray to select nine representative mixture rays from the system, the toxicities of six chemicals and nine rays at two exposure times on MTL-2 were determined, and toxicological interactions were systematically analyzed. Thus, the toxicity information of individual chemicals and various mixture rays in different genetically modified strains was obtained, and the toxicity interactions between different genetically modified strains were revealed, which provided a reference for mixture risk assessment.

\section{Methods \\ Test chemicals}

Two substituted phenols, 4-chlorophenol (4-CP) and 4-nitrophenol (4-NP), two pesticides, dichlorvos (DIC) and glyphosate (GLY), and two ionic liquids, 1-butylpyridinium chloride ([bpy]Cl) and 1-butylpyridinium bromide ([bpy]Br), were selected as mixture components to construct a six-component mixture system. DIC, GLY, [bpy] $\mathrm{Cl}$ and [bpy] $\mathrm{Br}$ were purchased from Sigma (USA), and 4-CP and 4-NP were purchased from CATO (USA). All solutions were prepared with Milli-Q water and stored at $4{ }^{\circ} \mathrm{C}$ before testing.

\section{Nematode culture}

The genetically modified C. elegans, strain CL2120 containing the mtl-2::GFP-linked reporter (MTL-2), was a generous gift from Prof. DaYong Wang of Southeast University (Nanjing, China), and the wild-type strain $\mathrm{N} 2$ (N2) and their food, E. coli OP50, were originally obtained from the Institute of Medicine of Tongji University (Shanghai, China), for use in this experiment. The detailed process of age synchronization, E. coli OP50 culture, nematode culture, subculture, blank and treatment group design, and lethality autoscaling are the same as the literatures $[20,35]$.

\section{Lethal toxicity test}

Age-synchronized nematodes were acquired so that the nematodes were at the same growth starting point, until the nematodes grew to the L4 stage for toxicity exposure. In the experiment, 96-well microplates were used as exposure vectors, and each well corresponded to one concentration. 
Each chemical treatment included 12 concentration gradients, and each concentration consisted of four parallel controls. Each plate included 6 blank controls $(100 \mu \mathrm{L}$ of ultrapure water and $100 \mu \mathrm{L}$ of nematode diluent). A total of $200 \mu \mathrm{L}$ of exposure solution (treatment group: $100 \mu \mathrm{L}$ of chemical solution and $100 \mu \mathrm{L}$ dilution of L4 C. elegans medium) and $15 \mathrm{~L} 4$ larvae were added into each well. To explore the effects of different exposure times on lethality, we used two different exposure times of 12 and $24 \mathrm{~h}$ in the absence of food in this work.

\section{Mixture design and curve fitting}

Notably, there are countless mixture rays with different concentration ratios in the six-component mixture system (SM) of [bpy]Br, [bpy]Cl, 4-CP, 4-NP, DIC, and GLY. Therefore, it is impossible to experimentally test the toxicities of all mixture rays and select representative mixture rays by means of optimal experimental designs, such as UD-Ray $[15,16]$. The uniform table $\mathrm{U}_{9}\left(9^{6}\right)$, where superscript 6 refers to components that have nine concentration levels $\left(\mathrm{EC}_{5}, \mathrm{EC}_{10}, \mathrm{EC}_{15}, \mathrm{EC}_{20}, \mathrm{EC}_{25}, \mathrm{EC}_{30}, \mathrm{EC}_{35}, \mathrm{EC}_{40}\right.$, and $\left.\mathrm{EC}_{45}\right)$ and the subscript 9 refers to nine representative rays (SM$\mathrm{Ri}, \mathrm{i}=1,2,3, \ldots, 9)$ [8], was used to design the basic concentration compositions (BCCs) of nine mixture rays (see Additional file 1: Table S1). The mixture ratios ( $p$ ) of components in various mixture rays calculated by the BCCs are listed in Table 1. For each mixture ray, 12 different concentration levels were specified by the fixed ratio ray design (FRRD) procedure [8].

To obtain the concentration-effect relationship of single components and mixture rays, the toxicity data at different concentrations were fitted to the Weibull function with two parameters (location $\alpha$ and shape $\beta$ ) by nonlinear least squares $[11,13]$ :

$$
\mathrm{X}=1-\exp \left(-\exp \left(\alpha+\beta \log _{10}(\mathrm{c})\right)\right),
$$

where $x$ is the lethality to $C$. elegans and $c$ the concentration of a single component or that of the mixture ray.

\section{Identification of toxicological interactions}

In this study, the CA model $[8,37]$ and improved combination index $\left(\mathrm{CI}_{\text {imp }}, 95 \%\right.$ observation-based confidence intervals (OCIs) were introduced into the combination index) [38] were used to qualitatively and quantitatively evaluate the toxicological interactions in the six-component mixture system. If the toxicity predicted by the CA model is located between the confidence intervals of experimental toxicity or the numerical value of 1 is located between the OCIs of $\mathrm{CI}_{i m p}$, the toxicity interaction of the mixture is additive (ADD). Synergistic (SYN) action refers to a toxicity predicted by CA that is less than the lower limit of the OCIs of experimental toxicity or by an upper confidence limit of $\mathrm{CI}_{i m p}$ of less than 1 . Antagonistic (ANT) action refers to a toxicity predicted by CA that is larger than the upper limit of the OCIs of experimental toxicity or by a lower confidence limit of $\mathrm{CI}_{\text {imp }}$ of larger than 1. The expression formulas of CA and CI shown in Eq. 2 [14] and Eq. 3 [38], respectively:

$$
\begin{aligned}
& \sum_{i=1}^{\mathrm{n}} \frac{c_{i}}{E C_{x, i}}=1, \\
& C I_{x}=\sum_{i=1}^{n} \frac{c_{i}}{E C_{x, i}},
\end{aligned}
$$

where $n$ is the number of mixture components, $E C_{x, i}$ is the concentration of the ith component that induces an $x$ percent effect when applied individually, and $c_{i}$ is the concentration of the ith component in the mixture that induces $x \%$ lethality.

The APTox (assessment and prediction for the toxicity of chemical mixtures) program developed in our laboratory [13] was used to perform all computations including autoscaling treatment, mixture design, CRC fitting, $\mathrm{CA}, \mathrm{CI}$ and their confidence intervals. The difference significance test (Origin Pro 7.5, Origin Lab Corp., USA)

\begin{tabular}{|c|c|c|c|c|c|c|}
\hline Ray & $P_{[\text {bpy }] \mathrm{Cl}}$ & $P_{[\mathrm{bpy}] \mathrm{Br}}$ & $P_{4-\mathrm{CP}}$ & $P_{4-\mathrm{NP}}$ & $P_{\text {DIC }}$ & $P_{\mathrm{GLY}}$ \\
\hline SM-R1 & $1.8740 \mathrm{E}-03$ & 7.7090E - 03 & $1.1800 E-01$ & $1.7600 E-01$ & $2.1745 E-01$ & $4.7897 E-01$ \\
\hline SM-R2 & $8.7420 E-03$ & $3.0065 E-02$ & $2.1394 \mathrm{E}-01$ & $8.9636 E-02$ & $1.6593 E-01$ & $4.9169 E-01$ \\
\hline SM-R3 & $2.1428 \mathrm{E}-02$ & $6.8045 E-02$ & $1.1924 \mathrm{E}-01$ & $2.3461 E-01$ & $9.8319 E-02$ & $4.5836 E-01$ \\
\hline SM-R4 & $4.2802 E-02$ & $1.3164 \mathrm{E}-01$ & $2.2037 E-01$ & $1.4233 E-01$ & $3.3179 E-02$ & $4.2968 E-01$ \\
\hline SM-R5 & $6.1113 E-02$ & $2.6830 E-03$ & $8.7740 E-02$ & $2.3992 E-01$ & $2.9103 E-01$ & $3.1751 E-01$ \\
\hline SM-R6 & $1.0365 E-01$ & $1.9670 E-02$ & $1.9245 E-01$ & $1.6920 E-01$ & $2.2613 E-01$ & $2.8889 E-01$ \\
\hline SM-R7 & $1.6371 \mathrm{E}-01$ & $5.4617 \mathrm{E}-02$ & 7.1703E- 02 & $3.1670 E-01$ & $1.5420 E-01$ & $2.3907 E-01$ \\
\hline SM-R8 & $2.3823 E-01$ & $1.1020 E-01$ & $1.9477 E-01$ & $2.2393 E-01$ & $7.5659 E-02$ & $1.5722 \mathrm{E}-01$ \\
\hline SM-R9 & $1.3761 \mathrm{E}-01$ & 7.9974E - 02 & $1.2421 E-01$ & $1.5122 \mathrm{E}-01$ & $1.8419 E-01$ & $3.2279 E-01$ \\
\hline
\end{tabular}
was carried out among the results from independent

Table 1 The mixture ratios $(p)$ of six components in the nine mixture rays to MTL-2 
experiments, and the statistic $(p)$ less than the significance levels given $(\alpha=0.05)$ was considered to be statistically significant.

\section{Results}

\section{Toxicities of six chemicals to MTL-2}

The CRCs of six chemicals at 12 and $24 \mathrm{~h}$ in MTL-2 (as well as those in N2 from our previous report [35]) were well fitted by the Weibull function (see Fig. 1). From Fig. 1, apart from the overlap of the two 4-CP CRCs in MTL-2 and N2 at $24 \mathrm{~h}$, the other CRCs in MTL-2 were located to the left side of those in N2 at the two exposure times, which implies that the toxicity on MTL- 2 at any concentration level at the same time is larger than that on $\mathrm{N} 2$. The results of the toxicity index $\left(p \mathrm{EC}_{50}\right)$ from Additional file 1: Table $\mathrm{S} 2$, validate this finding very well. On the other hand, the CRCs of the six components at $24 \mathrm{~h}$ were located to the left side those at $12 \mathrm{~h}$ in either MTL-2 or N2, which indicates that the toxicity of the component at $24 \mathrm{~h}$ is larger than that at $12 \mathrm{~h}$.

By using the $p \mathrm{EC}_{50}$ value on MTL-2 as the toxicity index, the most toxic compound at $12 \mathrm{~h}$ was GLY $\left(p \mathrm{EC}_{50}=2.758\right)$, and the least toxic compound was [bpy] $\mathrm{Br}$ (1.679). However, at $24 \mathrm{~h}$, the most toxic compound at $24 \mathrm{~h}$ was $[\mathrm{bpy}] \mathrm{Cl}$ (3.338), and the least toxic compound was GLY (2.796). The relative toxicity on MTL-2 at $24 \mathrm{~h}$ was [bpy]Cl (3.338) >4-CP (3.216) $>4$-NP (3.136) > [bpy] $\mathrm{Br}(3.082) \approx \mathrm{DIC}(3.016)>\mathrm{GLY}(2.796)$ while that at $12 \mathrm{~h}$ was GLY $\quad(2.758)>$ DIC $\quad(2.692)>4-\mathrm{CP} \quad(2.597) \approx 4-\mathrm{NP}$
(2.542) $>$ [bpy]Cl (1.747) $>$ [bpy] Br (1.679), which illustrates that the orders are different at the two times and indicates that the CRCs of the same compounds at different times are not completely parallel.

\section{Toxicities of six-component mixtures}

It was shown that the CRCs of nine mixture rays were monotonic S-shaped curves effectively fitted by the Weibull function (see Fig. 2). The fitted functions ( $\alpha$ and $\beta)$ and statistics ( $R^{2}$ and RMSE) of nine rays are listed in Table 2 together with the toxicity index $\left(p \mathrm{EC}_{50}\right)$. Different rays with different mixture ratios had different toxicities $\left(p \mathrm{EC}_{50}\right)$ at the same exposure times. The same mixture ray had different toxicities at different exposure times, and the toxicity at $24 \mathrm{~h}$ was larger than that at $12 \mathrm{~h}$, which is consistent with the results from individual components. On the other hand, the toxicity $\left(p \mathrm{EC}_{50}\right)$ of particular ray on MTL-2 was larger than that on N2, which implies that the risk of mixing is greater on the MTL-2 strain than that on the N2 strain.

From Table 2, by using the $p \mathrm{EC}_{50}$ as the toxicity index, the most toxic mixture ray at $12 \mathrm{~h}$ was the ray SM-R2 $\left(p \mathrm{EC}_{50}=3.164\right)$, and the least toxic one was ray SM-R5 (2.729). However, the most toxic mixture ray at $24 \mathrm{~h}$ was ray SM-R8 (3.568), and the least toxic was ray SM-R4 (3.108), which illustrates that the different rays with different mixture ratios have different toxicities in the same mixture system of the same components. That is, the toxicity of a multicomponent mixture (in fact, a mixture
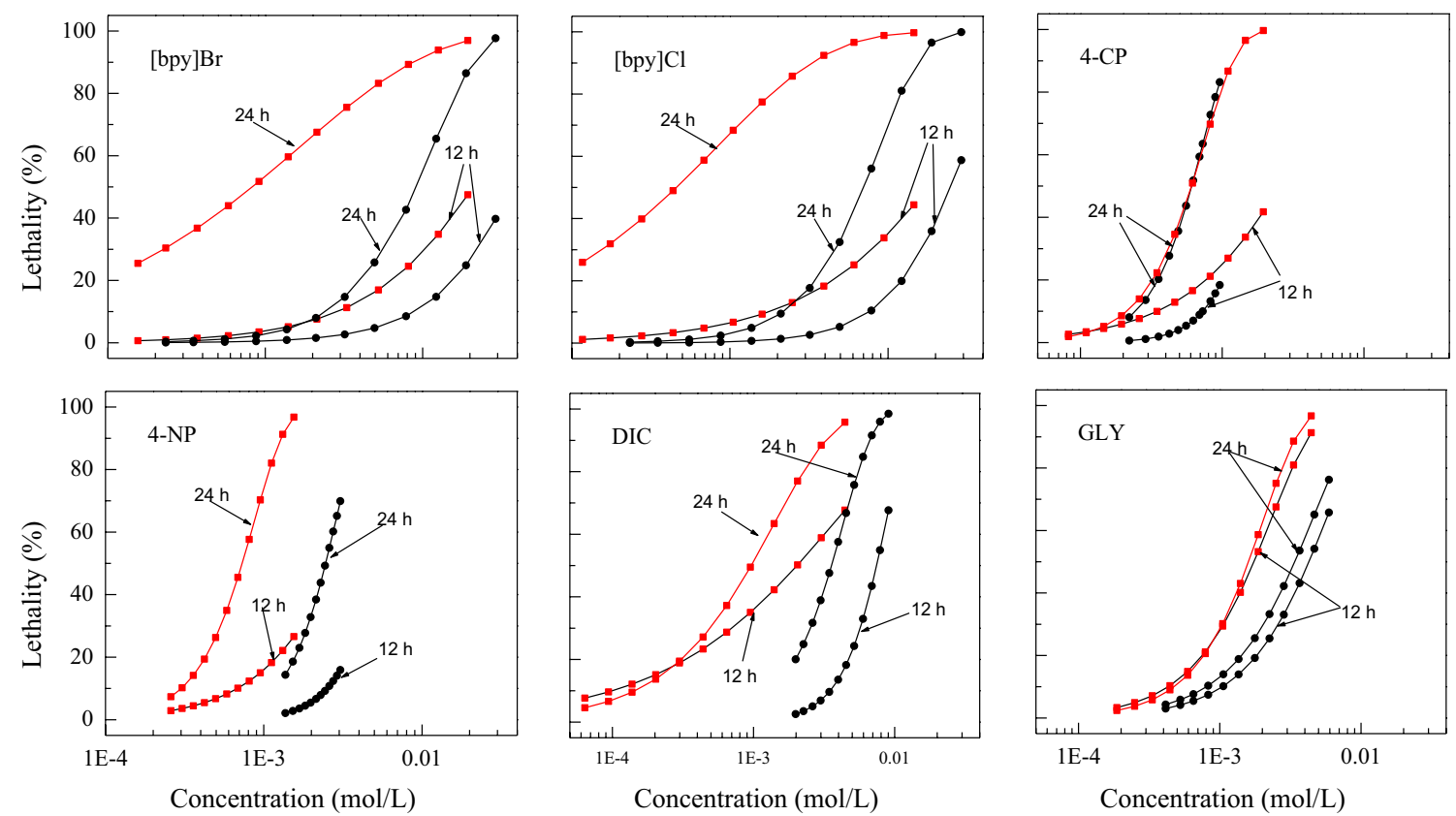

Fig. 1 The fitted concentration-response curves of six chemicals on N2 (black) and MTL-2 (red) 

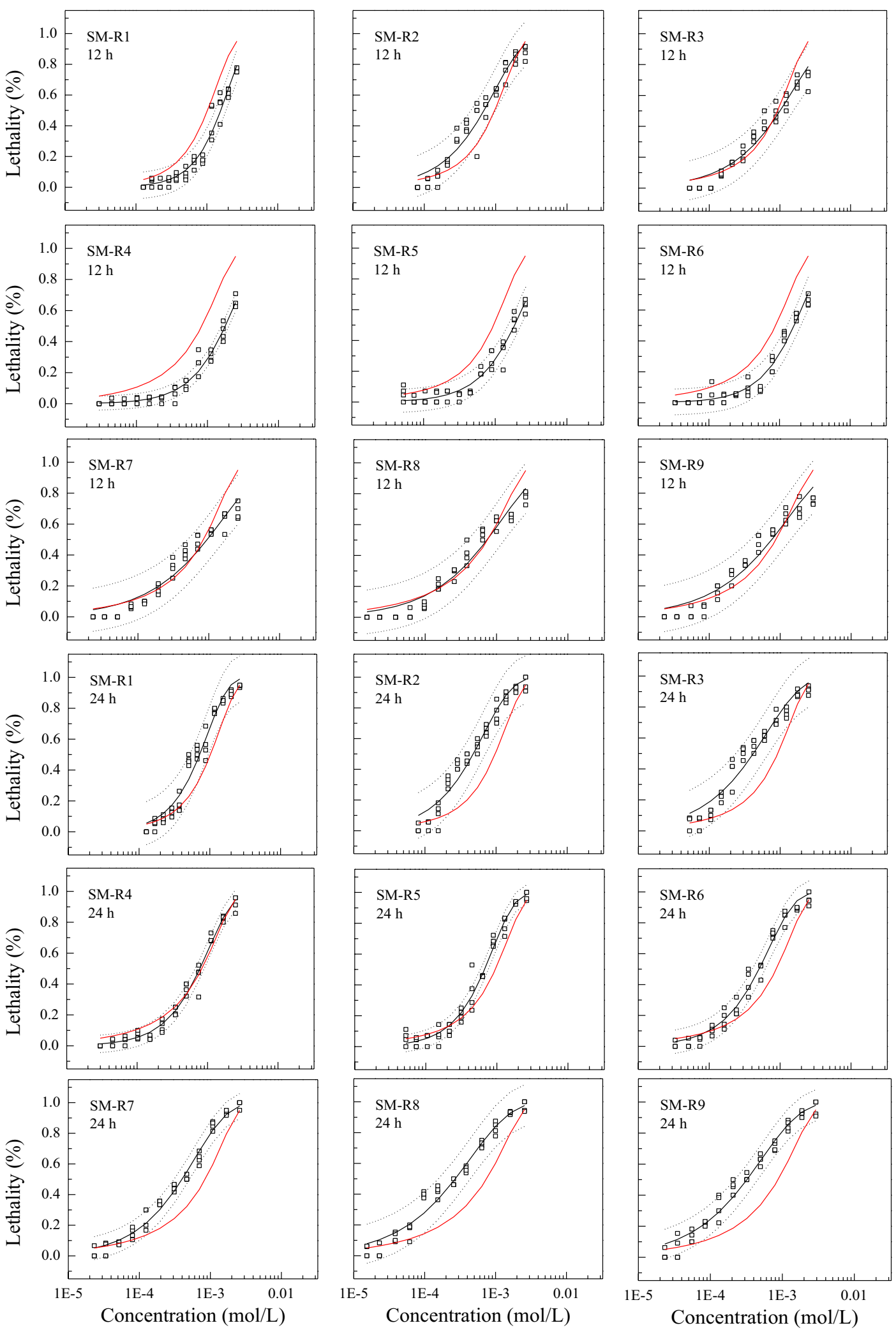

Fig. 2 The concentration-response curves (CRCs) of nine rays where open square refers to the experimental toxicities, the black solid lines to the CRCs fitted by Weibull functions, the black dashed lines to $95 \%$ observation-based confidence intervals, and the red lines to the CRCs predicted by concentration addition 
Table 2 The regression coefficients ( $\alpha$ and $\beta$ in the Weibull function), fitting statistics ( $R^{2}$ and RMSE), and $p E C_{50}$ values of nine mixture rays on MTL-2 at 12 and $24 h$

\begin{tabular}{|c|c|c|c|c|c|c|c|c|}
\hline Mixture ray & Time (h) & $E_{\text {max, exp }}$ & $a$ & $\beta$ & $R^{2}$ & RMSE & $P E C_{50}$ & $P E C_{50}^{*}$ \\
\hline \multirow[t]{2}{*}{ SM-R1 } & 12 & 0.7778 & 9.70 & 3.59 & 0.9819 & 0.0349 & 2.804 & 2.294 \\
\hline & 24 & 0.9892 & 10.04 & 3.31 & 0.9758 & 0.0555 & 3.144 & 2.559 \\
\hline \multirow[t]{2}{*}{ SM-R2 } & 12 & 0.9167 & 7.10 & 2.36 & 0.9725 & 0.0522 & 3.164 & 2.193 \\
\hline & 24 & 1.0000 & 7.10 & 2.36 & 0.9703 & 0.0577 & 3.321 & 2.401 \\
\hline \multirow[t]{2}{*}{ SM-R3 } & 12 & 0.7500 & 5.69 & 2.02 & 0.9654 & 0.0501 & 2.998 & 2.111 \\
\hline & 24 & 0.9375 & 6.22 & 1.95 & 0.9652 & 0.0576 & 3.378 & 2.408 \\
\hline \multirow[t]{2}{*}{ SM-R4 } & 12 & 0.7083 & 7.58 & 2.89 & 0.9920 & 0.0184 & 2.750 & 2.018 \\
\hline & 24 & 0.9583 & 8.46 & 2.84 & 0.9949 & 0.0230 & 3.108 & 2.277 \\
\hline \multirow[t]{2}{*}{ SM-R5 } & 12 & 0.6667 & 7.33 & 2.82 & 0.9776 & 0.0305 & 2.729 & 2.263 \\
\hline & 24 & 1.0000 & 9.60 & 3.14 & 0.9960 & 0.0224 & 3.174 & 2.578 \\
\hline \multirow[t]{2}{*}{ SM-R6 } & 12 & 0.7059 & 7.76 & 2.91 & 0.9776 & 0.0340 & 2.793 & 2.134 \\
\hline & 24 & 1.0000 & 8.37 & 2.65 & 0.9933 & 0.0306 & 3.297 & 2.417 \\
\hline \multirow[t]{2}{*}{ SM-R7 } & 12 & 0.7500 & 4.68 & 1.67 & 0.9558 & 0.0556 & 3.022 & 2.156 \\
\hline & 24 & 1.0000 & 6.77 & 2.10 & 0.9924 & 0.0299 & 3.398 & 2.407 \\
\hline \multirow[t]{2}{*}{ SM-R8 } & 12 & 0.8125 & 5.15 & 1.76 & 0.9638 & 0.0567 & 3.134 & - \\
\hline & 24 & 1.0000 & 5.77 & 1.72 & 0.9778 & 0.0499 & 3.568 & 2.354 \\
\hline \multirow[t]{2}{*}{ SM-R9 } & 12 & 0.7692 & 4.84 & 1.67 & 0.9581 & 0.0594 & 3.118 & 2.116 \\
\hline & 24 & 1.0000 & 5.92 & 1.80 & 0.9877 & 0.0372 & 3.493 & 2.318 \\
\hline
\end{tabular}

The values of $p \mathrm{EC}_{50}$ of nine mixture rays on $\mathrm{N} 2$ (Li et al. [35])

system) [8] has diversity, which implies that only the toxicity of the mixture ray with a specific mixture ratio can be compared with the toxicity of the same component.

\section{Evaluation of toxicity interactions}

\section{Qualitative evaluation of toxicity interaction}

Various toxicity interactions in mixtures were qualitatively evaluated by the concentration addition (CA) model [39-42]. The CRCs predicted by the CA model are also shown in Fig. 2. From Fig. 2, at $12 \mathrm{~h}$, the CRCs of three rays, SM-R4, SM-R5 and SM-R6, predicted by CA are located on the left side of the upper limits of the OCIs, which illustrates the antagonistic interactions (ANT) interactions in the mixture rays, while the predicted CRCs of the other six rays were mostly located between the OCIs, representing additive (ADD) actions. However, at $24 \mathrm{~h}$, the predicted CRCs of six rays, SM-R2, SM-R3, SM-R6, SM-R7, SM-R8 and SM-R9, were located on the right side of the lower limits of OCIs, which illustrates the synergistic (SYN) interactions in the mixture rays, while the predicted CRCs of the other rays were mostly located between the OCIs, representing additive (ADD) actions.

\section{Quantitative evaluation of toxicity interaction}

To quantitatively describe the toxicity interactions in chemical mixtures, the combination index (CI) $[20,38]$ was used, and the interaction results are shown in Fig. 3.

It can be seen from Fig. 3 that at $12 \mathrm{~h}$ of exposure, three rays SM-R4, SM-R5 and SM-R6 at most concentration levels exhibited obvious antagonistic (ANT) interactions, the SM-R1 ray displayed slight ANT, and the SM-3, SM-R7, SM-R8 and SM-R9 rays are showed additive (ADD) actions, whereas the SM-3 and SM-R7 rays at high concentration levels synergistic (SYN) interactions. In other words, half of the mixture rays had ANT interactions and the other half had ADD actions, which is different from the major ANT interactions in C. elegans with modified SOD-3 [35] and from the SYN interactions of most mixture rays and the ADD action of a few rays in wild-type N2 C. elegans [20].

At $24 \mathrm{~h}$ of exposure, although the SM-R4 ray exhibited ADD interactions in MTL-2, the other eight rays mainly displayed SYN interactions, which is different from the major ADD actions of mixtures in both the SOD- 3 and N2 strains.

Integrated with the toxicity interactions at the two exposure times, it can be concluded that the toxicity interaction of the six-component mixture on MTL-2 changes from ANT/ADD to primarily SYN with exposure time and that the toxicity interaction is timedependent. This transition from ANT/ADD to SYN 

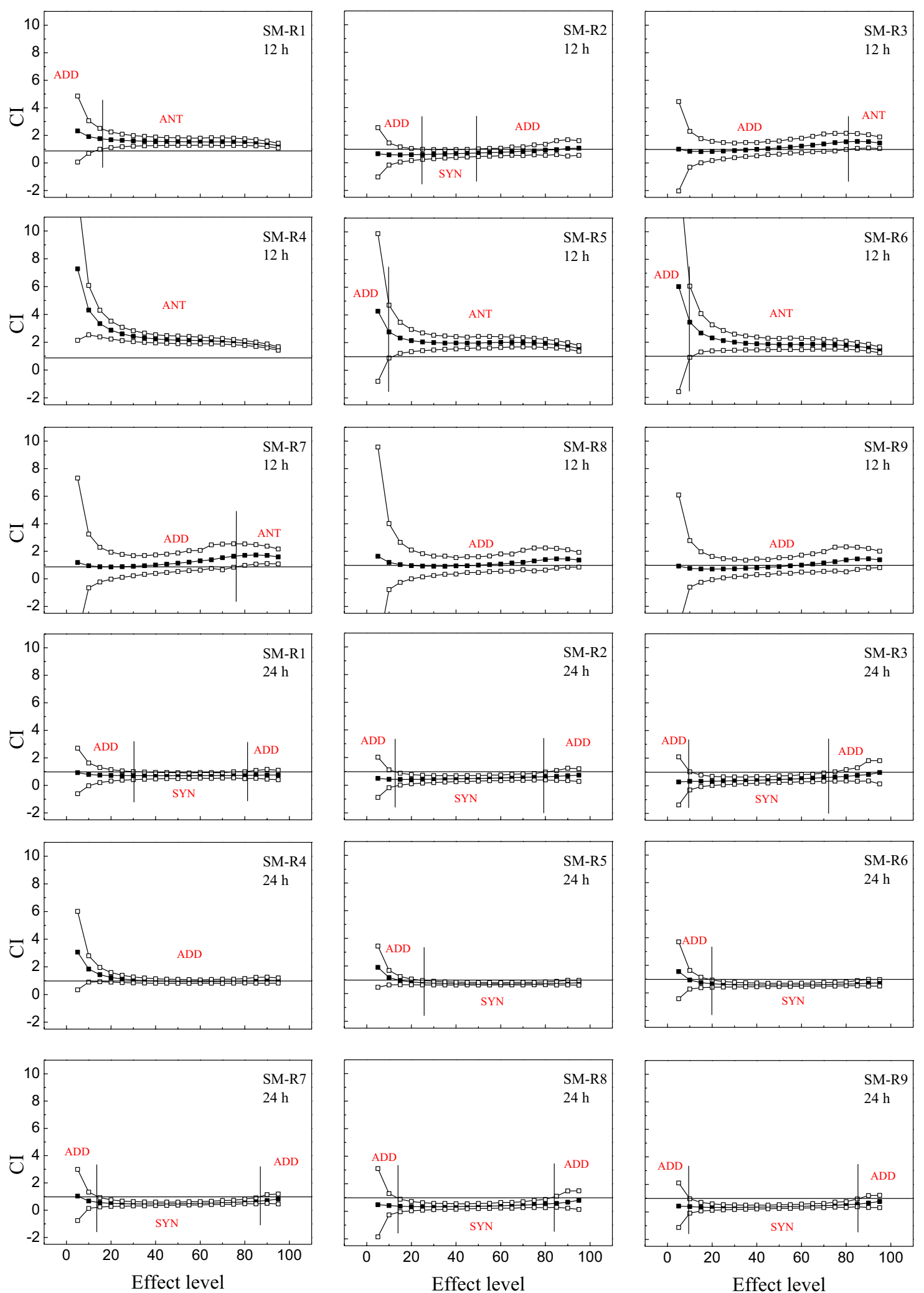

Fig. 3 Plots of the combination indies (Cls) vs. effect levels at two exposure times where filled square box refers to Cls, open square box to 95\% observation-based confidence intervals of Cls. ADD additive action, ANT antagonism, and SYN synergism 
with the extension of exposure time is different from the transition from ANT to ADD in SOD-3 animals [35] and from the transition from SYN to ADD in N2 [20].

\section{Discussion}

\section{Showing the different toxicities of six chemicals} to different strains

The results showed that there were differences in both single compound and six-component mixture toxicity between wild-type and genetically modified C. elegans. Considering previous studies, in a mixture system composed of the same compounds, different nematode strains showed different time-dependent toxicities with the extension of exposure time (ANT/ADD to SYN in MTL-2, ANT to ADD in SOD-3 [35] and SYN to ADD in N2 [20]). These differences indicate that genetic modification has an effect on organisms to some extent. Therefore, we should pay special attention to the selection of test organisms in the study of toxicity of mixtures.

The literature about the different toxicological interactions on the same organism sharing one toxic endpoint at the same exposure time is limited. The genetically modified daf-16 CF1038 strain had a shortened lifespan (i.e., approximately $70 \%$ of the wild-type strain lifespan) under exposure to several individual chemicals, and weakly increased lethality of $\mathrm{N} 2$ animals (or weak tolerance of the daf-16 strain) was detected in the presence of $3.1 \mu \mathrm{M}$ dichlorvos [43]. The genetically modified $m t l-2$ and $m e v-$ 1 strains were more sensitive to AgNP exposure than were wild-type [44]. Other research has reported that compared with wild-type, the genetically modified $m t l$ 1 and $m t l-2$ strains had increased sensitivity to stress or toxic reactions [45].

A similar conclusion was obtained in other studies that reported the related toxicity data of the same chemical in different model organisms [46]. Under the current environmental concentration of chlortetracycline $(0.5 \mathrm{mg} / \mathrm{L})$, the percent viability of the initial bacterial inoculum was reduced to $0.22 \%$ and $0.08 \%$ in Gram-positive Bacillus thuringiensis and Gram-negative Enterobacter aerogenes, respectively [47]. This also illustrates that the same compound can have different toxicities in different strains of the same organism even when the toxicity endpoint is the same. Either way, the effect of genetic modification in the same model organisms or toxic endpoint should be considered.

\section{Concentration-response relationships of mixtures}

Normally, a lower concentration range is more suitable for the real environment; moreover, organisms or humans are exposed to complex mixtures of low-dose compounds rather than single compounds [3, 48]. With the concentration-response relationship, we can find the toxic effect of the concentration corresponding to the actual environment on the tested organisms, and we can preliminarily determine the harmfulness of pollutants mixtures to the organisms in the actual environment. In the cases of this study, the same organism with the same toxic endpoint showed clearly different toxicological interactions of mixtures only because of genetic modification. In addition, the toxicity of the mixture between different genetically modified strains was also different. The same mixture had different toxicity interactions in different strains of organisms, and the toxicity interactions of the mixture were time dependent [20, 35]. Therefore, risk assessment based on mixture toxicity is more important than that based on individual toxicity, and risk assessment based on genetically modified organisms should be more cautious than that based on normal organisms. Otherwise, these differences may lead to inaccurate evaluation results.

\section{AOP may solve the mixture toxicological interactions}

One of the possible mechanisms that we think may have triggered these changes is changing the binding mode of the original protein and increasing the protein-protein interface with the intervention of GFP. It is assumed that $\mathrm{CA}$ is based on the premise that all components have similar mechanisms of action (MOA) $[49,50]$. However, how do we define a similar MOA? Except for the selection of reference models, there is a shortage of explanations for the relevant mechanism of mixture toxicological interaction. Moreover, the mechanism of toxicity of a single substance may not be suitable for mixtures. The exploration of possible mechanisms of mixture toxicity is a difficult and complex process. It seems that we could solve this issue by researching the adverse outcome pathway (AOP) - a pragmatic and popular tool in toxicology.

AOP is a conceptual construct that integrates existing knowledge concerning the pathway of causal linkages between an initiating molecular event and a final adverse effect at the individual or population levels [51-54]. In addition, AOP can provide a more important starting point than MOA when planning an assessment or predictions regarding the toxicity effects of a mixture on the environment [55]. Because the related pathway is a not known, there is no means to explain the toxic mechanism even if the initiating molecular events and the final results are the same in N2 and MTL-2.

\section{Conclusions}

In this study, we combined two C. elegans strain analyses, $\mathrm{CI}_{\text {imp }}$ and CA, to evaluate the nontarget time-dependent toxicities of a six-component mixture system. The results showed that although the toxicity of 4-chlorophenol on MTL-2 was not significantly different from that on N2, 
the toxicities of the other five chemicals on MTL-2 were greater than those on N2. The toxicities of six single chemicals and nine mixture rays on MTL- 2 increased with time, which is consistent with those on N2 and on the SOD-3. At $12 \mathrm{~h}$, half of the toxicological interactions of various mixtures on MTL-2 were a half antagonistic (ANT), and half were additive (ADD), while at $24 \mathrm{~h}$, the interactions were mainly synergistic (SYN). The toxicological interactions of various mixtures on MTL-2 changed from ANT/ADD to primarily SYN with time, which is different from the change from ANT to ADD in the SOD-3 strain and different from the change from SYN to ADD in N2. Therefore, the toxicities and toxicity interactions of chemical mixtures on different Caenorhabditis elegans strains are different. It is necessary to examine the effect of genetic modification on the toxicological interaction of mixtures to avoid underestimating or overestimating mixture risk.

\section{Supplementary information}

Supplementary information accompanies this paper at https://doi. org/10.1186/s12302-020-00337-2.

Additional file 1: Additional tables

\section{Abbreviations}

MTL-2: Transgenic strain mt-2::GFP; N2: Wild-type strain; FRRD: Fixed ratio ray design; EECR: Equivalent toxicity/effect concentration ratio; UD-Ray: Uniform design-based ray design; C. elegans: Caenorhabditis elegans; GFP: Green fluorescent protein; HepG2: Hepatocarcinoma; 4-CP: 4-Chlorophenol; 4-NP. 4-Nitrophenol; DIC: Dichlorvos; GLY: Glyphosate; [bpy]Cl: 1-Butylpyridinium chloride; [bpy]Br: 1-Butylpyridinium bromide; SM: Six-component mixture system; BCCs: Basic concentration compositions; CA: Concentration addition; $\mathrm{Cl}_{\text {imo: }}$ Improved combination index; OCls: 95\% observation-based confidence intervals; ADD: Additive; SYN: Synergistic; ANT: Antagonistic; CRC: Concentration-response curve; AOP: Adverse outcome pathway.

\section{Acknowledgements}

We are thankful to the National Key Research and Development Program of China (2018YFC1603003) and National Natural Science Foundation of China $(21677113,21437004)$ for their financial support.

\section{Authors' contributions}

$\mathrm{PH}$ and $\mathrm{KL}$ contributed to the experimental studies, data acquisition, analysis, manuscript preparation, and editing. YQX and ZJW contributed to the experimental discussion and approved the final manuscript. SSL provided guidance in writing papers and experiments. All authors read and approved the final manuscript.

\section{Funding}

The National Key Research and Development Program of China (2018YFC1603003) and the National Natural Science Foundation of China (21677113, 21437004)

\section{Availability of data and materials}

The authors declare that all data supporting the findings of this study are available in the article and its supplementary information files.

Ethics approval and consent to participate

Not applicable.
Consent for publication

Not applicable.

\section{Competing interests}

The authors declare no conflicts of interest.

\section{Author details}

${ }^{1}$ Key Laboratory of Yangtze River Water Environment, College of Environmental Science and Engineering, Ministry of Education, Tongji University, 1239 Siping Road, Shanghai 200092, P. R. China. ${ }^{2}$ Shanghai Institute of Pollution Control and Ecological Security, Shanghai 200092, P. R. China. ${ }^{3}$ State Key Laboratory of Pollution Control and Resource Reuse, College of Environmental Science and Engineering, Tongji University, Shanghai 200092, P. R. China.

Received: 14 October 2019 Accepted: 23 March 2020

Published online: 16 April 2020

\section{References}

1. Rowlands JC, Sander M, Bus JS, FutureTox Organizing C (2014) FutureTox: building the road for 21st century toxicology and risk assessment practices. Toxicol Sci 137:269-277. https://doi.org/10.1093/toxsci/kft252

2. Scott GR, Sloman KA (2004) The effects of environmental pollutants on complex fish behaviour: integrating behavioural and physiological indicators of toxicity. Aquat Toxicol 68:369-392. https://doi.org/10.1016/j. aquatox.2004.03.016

3. Jonker MJ, Svendsen C, Bedaux JJM, Bongers M, Kammenga JE (2005) Significance testing of synergistic/antagonistic, dose level-dependent, or dose ratio-dependent effects in mixture dose-response analysis. Environ Toxicol Chem 24:2701-2713. https://doi.org/10.1897/04-431r.1

4. Yu M, Liu S, Wang M, Chen F, Tang H (2014) Mixture toxicities of three pesticides having different time-toxicity profiles. Chin J Chem 32:545-552. https://doi.org/10.1002/cjoc.201400133

5. Kretschmann A, Gottardi M, Dalhoff K, Cedergreen N (2015) The synergistic potential of the azole fungicides prochloraz and propiconazole toward a short alpha-cypermethrin pulse increases over time in Daphnia magna. Aquat Toxicol 162:94-101. https://doi.org/10.1016/j.aquatox.2015.02.011

6. Syberg K, Binderup M-L, Cedergreen N, Rank J (2015) Mixture genotoxicity of 2,4-dichlorophenoxyacetic acid, acrylamide, and maleic hydrazide on human Caco-2 cells assessed with comet assay. J Toxicol Environ Health Part Curr Issues 78:369-380. https://doi.org/10.1080/15287 394.2014.983626

7. Zheng Q-F, Ju Z, Liu S-S (2019) Combined toxicity of dichlorvos and its metabolites to Vibrio qinghaiensis sp.-Q67 and Caenorhabditis elegans. Acta Chim Sinica 77:1008-1016. https://doi.org/10.6023/a19060197

8. Liu S-S, Xiao Q-F, Zhang J, Yu M (2016) Uniform design ray in the assessment of combined toxicities of multi-component mixtures. Sci Bull 61:52-58. https://doi.org/10.1007/s11434-015-0925-6

9. Zhang J, Liu L, Ren L, Feng W, Lv P, Wu W, Yan Y (2017) The single and joint toxicity effects of chlorpyrifos and beta-cypermethrin in zebrafish (Danio rerio) early life stages. J Hazard Mater 334:121-131. https://doi. org/10.1016/j.jhazmat.2017.03.055

10. Wang YH, Wu SG, Chen JE, Zhang CP, Xu ZL, Li G, Cai LM, Shen WF, Wang $Q$ (2018) Single and joint toxicity assessment of four currently used pesticides to zebrafish (Danio rerio) using traditional and molecular endpoints. Chemosphere 192:14-23. https://doi.org/10.1016/j.chemospher e.2017.10.129

11. Liu S (2017) Assessment and prediction of toxicity of chemical mixtures. Sci Press, Beijing, p 155

12. Liu S-S, Li K, Li T, Qu R (2016) Comments on "The synergistic toxicity of the multi chemical mixtures: implications for risk assessment in the terrestrial environment". Environ Int 94:396-398. https://doi.org/10.1016/j.envin t.2016.04.038

13. Liu S, Zhang J, Zhang Y, Qin L (2012) APTox: assessment and prediction on toxicity of chemical mixtures. Acta Chim Sinica 70:1511-1517. https:// doi.org/10.6023/a12050175

14. Liu S, Liu L, Chen F (2013) Application of the concentration addition model in the assessment of chemical mixture toxicity. Acta Chim Sinica 71:1335-1340. https://doi.org/10.6023/a13040355 
15. Fan Y, Liu S-S, Qu R, Li K, Liu H-L (2017) Polymyxin B sulfate inducing timedependent antagonism of the mixtures of pesticide, ionic liquids, and antibiotics to Vibrio qinghaiensis sp.-Q67. Rsc Adv 7:6080-6088. https:// doi.org/10.1039/c6ra25843c

16. Xu Y-Q, Liu S-S, Fan Y, Li K (2018) Toxicological interaction of multi-component mixtures to Vibrio qinghaiensis sp.-Q67 induced by at least three components. Sci Total Environ 635:432-442. https://doi.org/10.1016/j. scitotenv.2018.04.023

17. Shen L, Xiao J, Ye H, Wang D (2009) Toxicity evaluation in nematode Caenorhabditis elegans after chronic metal exposure. Environ Toxicol Pharmacol 28:125-132. https://doi.org/10.1016/j.etap.2009.03.009

18. Wang D, Liu P, Yang Y, Shen L (2010) Formation of a combined $\mathrm{Ca} / \mathrm{Cd}$ toxicity on lifespan of nematode Caenorhabditis elegans. Ecotoxicol Environ Saf 73:1221-1230. https://doi.org/10.1016/j.ecoenv.2010.05.002

19. Wang $D$, Xing $X(2010)$ Pre-treatment with mild UV irradiation suppresses reproductive toxicity induced by subsequent cadmium exposure in nematodes. Ecotoxicol Environ Saf 73:423-429. https://doi.org/10.1016/j. ecoenv.2009.12.014

20. Feng L, Liu S-S, Li K, Tang H-X, Liu H-L (2017) The time-dependent synergism of the six-component mixtures of substituted phenols, pesticides and ionic liquids to Caenorhabditis elegans. J Hazard Mater 327:11-17. https://doi.org/10.1016/j.jhazmat.2016.12.031

21. Tsien RY (1998) The green fluorescent protein. Annu Rev Biochem 67:509-544. https://doi.org/10.1146/annurev.biochem.67.1.509

22. $\mathrm{Ma} \mathrm{H}$, Glenn TC, Jagoe CH, Jones KL, Williams PL (2009) A transgenic strain of the nematode Caenorhabditis elegans as a biomonitor for heavy metal contamination. Environ Toxicol Chem 28:1311-1318. https://doi. org/10.1897/08-496.1

23. Anbalagan C, Lafayette I, Antoniou-Kourounioti M, Gutierrez C, Rodriguez Martin J, Chowdhuri DK, De Pomerai DI (2013) Use of transgenic GFP reporter strains of the nematode Caenorhabditis elegans to investigate the patterns of stress responses induced by pesticides and by organic extracts from agricultural soils. Ecotoxicology 22:72-85. https://doi. org/10.1007/s10646-012-1004-2

24. Fitsanakis VA, Negga R, Hatfield HE (2014) Caenorhabditis elegans as a model for biomarkers of diseases and toxicities. Biomarkers in Toxicology, Gupta RC (ed). 113128

25. Tang H-X, Liu S-S, Li K, Feng L (2016) Combining the uniform designbased ray procedure with combination index to investigate synergistic lethal toxicities of ternary mixtures on Caenorhabditis elegans. Anal Methods 8:4466-4472. https://doi.org/10.1039/c6ay00582a

26. Tejeda-Benitez L, Flegal R, Odigie K, Olivero-Verbel J (2016) Pollution by metals and toxicity assessment using Caenorhabditis elegans in sediments from the Magdalena River, Colombia. Environ Pollut 212:238-250. https:// doi.org/10.1016/j.envpol.2016.01.057

27. Tejeda-Benitez L, Noguera-Oviedo K, Aga DS, Olivero-Verbel J (2018) Toxicity profile of organic extracts from Magdalena River sediments. Environ Sci Pollut Res 25:1519-1532. https://doi.org/10.1007/s11356-017-0364-9

28. Wang D, Shen L, Wang Y (2007) The phenotypic and behavioral defects can be transferred from zinc-exposed nematodes to their progeny. Environ Toxicol Pharmacol 24:223-230. https://doi.org/10.1016/j. etap.2007.05.009

29. Wang Y, Xie W, Wang D (2007) Transferable properties of multi-biological toxicity caused by cobalt exposure in Caenorhabditis elegans. Environ Toxicol Chem 26:2405-2412. https://doi.org/10.1897/06-646r1.1

30. McVey KA, Snapp IB, Johnson MB, Negga R, Pressley AS, Fitsanakis VA (2016) Exposure of $C$. elegans eggs to a glyphosate-containing herbicide leads to abnormal neuronal morphology. Neurotoxicol Teratol 55:23-31. https://doi.org/10.1016/..ntt.2016.03.002

31. Lenz KA, Pattison C, Ma H (2017) Triclosan (TCS) and triclocarban (TCC) induce systemic toxic effects in a model organism the nematode Caenorhabditis elegans. Environ Pollut 231:462-470. https://doi.org/10.1016/j. envpol.2017.08.036

32. Yang R, Ren M, Rui Q, Wang D (2016) A mir-231-Regulated protection mechanism against the toxicity of graphene oxide in nematode Caenorhabditis elegans. Sci Rep. https://doi.org/10.1038/srep32214

33. Li P, Xu T, Wu S, Lei L, He D (2017) Chronic exposure to graphene-based nanomaterials induces behavioral deficits and neural damage in Caenorhabditis elegans. J Appl Toxicol 37:1140-1150. https://doi.org/10.1002/ jat.3468
34. O'Donnell B, Huo L, Polli JR, Qiu L, Collier DN, Zhang B, Pan X (2017) ZnO nanoparticles enhanced germ cell apoptosis in Caenorhabditis elegans, in comparison with ZnCl2. Toxicol Sci 156:336-343. https://doi.org/10.1093/ toxsci/kfw258

35. Li K, Xu Y-Q, Feng L, Liu S-S (2018) Assessing the influence of the genetically modified factor on mixture toxicological interactions in Caenorhabditis elegans: comparison between wild type and a SOD type. Environ Pollut 242:872-879. https://doi.org/10.1016/j.envpol.2018.06.107

36. Freedman JH, Slice LW, Dixon D, Fire A, Rubin CS (1993) The novel metallothionein genes of Caenorhabditis elegans. Structural organization and inducible, cell-specific expression. J Biol Chem 268:2554-2564

37. Liu L, Liu S-S, Yu M, Zhang J, Chen F (2015) Concentration addition prediction for a multiple-component mixture containing no effect chemicals. Anal Methods 7:9912-9917. https://doi.org/10.1039/c5ay0 $1784 j$

38. Liu L, Liu S-S, Yu M, Chen F (2015) Application of the combination index integrated with confidence intervals to study the toxicological interactions of antibiotics and pesticides in Vibrio qinghaiensis sp.-Q67. Environ Toxicol Pharmacol 39:447-456. https://doi.org/10.1016/j.etap.2014.12.013

39. Ju Z, Liu S-S, Xu Y-Q, Li K (2019) Combined toxicity of 2,4-dichlorophenoxyacetic acid and its metabolites 2,4-dichlorophenol (2,4-DCP) on two nontarget organisms. Acs Omega 4:1669-1677. https://doi.org/10.1021/ acsomega.8b02282

40. Li T, Liu S-S, Qu R, Liu H-L (2017) Global concentration additivity and prediction of mixture toxicities, taking nitrobenzene derivatives as an example. Ecotoxicol Environ Saf 144:475-481. https://doi.org/10.1016/j. ecoenv.2017.06.044

41. Cedergreen N, Kudsk P, Mathiassen SK, Streibig JC (2007) Combination effects of herbicides on plants and algae: do species and test systems matter? Pest manage. Science 63:282-295. https://doi.org/10.1002/ ps. 1353

42. Syberg K, Elleby A, Pedersen H, Cedergreen N, Forbes VE (2008) Mixture toxicity of three toxicants with similar and dissimilar modes of action to Daphnia magna. Ecotoxicol Environ Saf 69:428-436. https://doi. org/10.1016/j.ecoenv.2007.05.010

43. Kurauchi M, Morise H, Eki T (2009) Using the nematode Caenorhabditis elegans daf-16 mutant to assess the lifespan toxicity of prolonged exposure to ecotoxic agents. J Health Sci 55:796-804. https://doi.org/10.1248/ jhs. 55.796

44. Meyer JN, Lord CA, Yang XY, Turner EA, Badireddy AR, Marinakos SM Chilkoti A, Wiesner MR, Auffan M (2010) Intracellular uptake and associated toxicity of silver nanoparticles in Caenorhabditis elegans. Aquat Toxicol 100:140-150. https://doi.org/10.1016/j.aquatox.2010.07.016

45. Swain SC, Keusekotten K, Baumeister R, Sturzenbaum SR (2004) C. elegans metallothioneins: new insights into the phenotypic effects of cadmium toxicosis. J Mol Biol 341:951-959. https://doi.org/10.1016/j. jmb.2004.06.050

46. Lipok J, Studnik H, Gruyaert S (2010) The toxicity of Roundup (R) 360 SL formulation and its main constituents: glyphosate and isopropylamine towards non-target water photoautotrophs. Ecotoxicol Environ Saf 73:1681-1688. https://doi.org/10.1016/j.ecoenv.2010.08.017

47. Pulicharla R, Das RK, Brar SK, Drogui P, Sarma SJ, Verma M, Surampalli RY, Valero JR (2015) Toxicity of chlortetracycline and its metal complexes to model microorganisms in wastewater sludge. Sci Total Environ 532:669-675. https://doi.org/10.1016/.jscitotenv.2015.05.140

48. Cedergreen N (2014) Quantifying synergy: a systematic review of mixture toxicity studies within environmental toxicology. PLoS ONE. https://doi. org/10.1371/journal.pone.0096580

49. Ohlsson A, Cedergreen N, Oskarsson A, Ulleras E (2010) Mixture effects of imidazole fungicides on cortisol and aldosterone secretion in human adrenocortical H295R cells. Toxicology 275:21-28. https://doi. org/10.1016/j.tox.2010.05.013

50. Bliss Cl (1939) The toxicity of poisons applied jointly. Ann J Appl Biol 26:585-615

51. Ankley GT, Bennett RS, Erickson RJ, Hoff DJ, Hornung MW, Johnson RD, Mount DR, Nichols JW, Russom CL, Schmieder PK, Serrrano JA, Tietge JE, Villeneuve DL (2010) Adverse outcome pathways: a conceptual framework to support ecotoxicology research and risk assessment. Environ Toxicol Chem 29:730-741. https://doi.org/10.1002/etc.34 
52. Vinken M (2013) The adverse outcome pathway concept: a pragmatic tool in toxicology. Toxicology 312:158-165. https://doi.org/10.1016/j. tox.2013.08.011

53. Villeneuve DL, Crump D, Garcia-Reyero N, Hecker M, Hutchinson TH, LaLone CA, Landesmann B, Lettieri T, Munn S, Nepelska M, Ottinger MA, Vergauwen L, Whelan M (2014) Adverse outcome pathway (AOP) development I: strategies and principles. Toxicol Sci 142:312-320. https://doi. org/10.1093/toxsci/kfu199

54. Baldrick P (2017) Pharmacokinetic and toxicology comparator testing of biosimilar drugs_assessing need. Regul Toxicol Pharmacol 86:386-391. https://doi.org/10.1016/j.yrtph.2017.04.010
55. Lopes S, Pinheiro C, Soares AMVM, Loureiro S (2016) Joint toxicity prediction of nanoparticles and ionic counterparts: simulating toxicity under a fate scenario. J Hazard Mater 320:1-9. https://doi.org/10.1016/j.jhazm at.2016.07.068

\section{Publisher's Note}

Springer Nature remains neutral with regard to jurisdictional claims in published maps and institutional affiliations.

\section{Submit your manuscript to a SpringerOpen ${ }^{\circ}$ journal and benefit from:}

- Convenient online submission

- Rigorous peer review

- Open access: articles freely available online

- High visibility within the field

- Retaining the copyright to your article

Submit your next manuscript at $\boldsymbol{\nabla}$ springeropen.com 